\title{
Automated Approximation of Lateral Ventricular Shape in Magnetic Resonance Images of Multiple Sclerosis Patients
}

\author{
Bernhard Sturm ${ }^{1}$, Dominik Meier ${ }^{2}$, and Elizabeth Fisher ${ }^{1}$ \\ ${ }^{1}$ Department of Biomedical Engineering / ND20, Lerner Research Institute, \\ The Cleveland Clinic Foundation, 9500 Euclid Avenue, Cleveland, Ohio 44195, U.S.A. \\ \{sturm, fisher\}@bme.ri.ccf.org \\ ${ }^{2}$ Center for Neurological Imaging, Brigham \& Women's Hospital, Harvard Medical School, \\ 221 Longwood Avenue- RFB 398, Boston, Massachusetts 02115 \\ meier@bwh.harvard.edu
}

\begin{abstract}
Active surfaces" or deformable models have been proposed for the segmentation of anatomic structures in MRI data. Such algorithms are dependent on a good initial approximation of the target shape. The purpose of this work was to develop a reliable method for automatic generation of a starting point for segmentation of the lateral ventricle. The algorithm uses a parametric representation of an average lateral ventricle, which is customized for each individual by modulating the parametric coefficients based on the brain parenchymal fraction. The method was developed with a training set of 6 healthy controls and 25 patients with multiple sclerosis, and tested on an additional set of 10 patients. Compared to the average ventricle, this new approach provided a closer approximation to the manually segmented ventricular shape in $81 \%$ of the cases in the training set and $100 \%$ of the additional test set.
\end{abstract}

\section{Introduction}

Automated segmentation of the lateral ventricles in MRI data would be beneficial for quantification of regional atrophy, and for ongoing efforts in structure driven anatomical labeling of the brain [1]. A successful segmentation algorithm would alleviate issues of operator variability and accelerate the current method. The improved precision provided by automated segmentation is of particular value in the study of neurodegenerative diseases, where morphological changes are subtle, and a high sensitivity is required for their detection.

Approaches for segmentation of anatomic structures include algorithms such as "active surfaces" or deformable models [2]-[7]. For successful and reproducible convergence, such algorithms typically require an initialization that represents a reasonably close approximation of the target shape. Little has been reported on the selection and evaluation of such initializations. Manual identification of a starting point provides a customized initialization but can be time-consuming and introduces observer variability. Initialization from average population models is automated but potentially limited by the variability of the target population. Population based models also allow the introduction of additional prior knowledge, such as statistics to limit the extent to 
which a model can deform [4]-[7]. However, such approaches often rely solely on the average population model as their starting point. We demonstrate in this work that additional information derived automatically from the individual case can be the basis for customizing an average population model and thus lead to a better approximation of the target shape. Such an approach has direct applications to automated segmentation algorithms based on deformable models.

The purpose of this study was to test an automatically generated shape approximation of the lateral ventricle in multiple sclerosis (MS) patients and normal healthy volunteers. We propose a parametric model of ventricular shape, linked to the brain parenchymal fraction (BPF), where BPF is a size-normalized estimate of whole brain atrophy [8]-[9]. BPF calculation is fully automated and readily available. The relationship between the coefficients of a parametric description and BPF can be used to extrapolate the shape of a ventricle for any individual based on the BPF. The concept is similar to that presented in [10] where the position of anatomical structures in the brain are predicted based on the location and parameterization of the head surface.

\section{Methods}

\subsection{Population}

MR images from $25 \mathrm{MS}$ patients and 6 healthy volunteers were selected to represent a sample of MS patients with varying disease type, duration and severity (13 relapsingremitting MS, 9 secondary progressive MS, and 3 primary progressive MS). These 31 cases formed the training set for the average ventricle shape. Images from 10 additional MS patients (6 relapsing-remitting, 4 secondary progressive) were selected for testing purposes.

\subsection{BPF Calculations}

BPF was determined automatically on each MRI image as described in [8]-[9]. An automated 3D segmentation algorithm was applied to identify brain tissue voxels and the smoothed outer surface of the brain. The algorithm calculated the brain volume (BV) while accounting for partial volume effects. The outer brain contour volume (OCV), i.e. the volume enclosed by the smoothed outer surface of the brain, is also determined. The BPF is then defined as:

$$
\mathrm{BPF}=\mathrm{BV} / \mathrm{OCV}
$$

$\mathrm{BPF}$ thus represents a normalized estimate of brain volume.

\subsection{Spherical Harmonic Parameterization of the Lateral Ventricle}

The left lateral ventricle of each case was segmented by manual tracing using customized software developed in IDL (Research Systems Incorporated, Boulder, U.S.A.). A parametric representation of each segmented left ventricle based on spherical harmonics was generated as described in [1], [11]. The Cartesian coordi- 
nates of uniformly sampled points on the surface of the ventricle can be homologically mapped onto the surface of a sphere. Spherical harmonics $Y_{1}^{\mathrm{m}}(\theta, \phi)$ were used as the basis functions for a parametric representation of the ventricular surface, according to:

$$
\begin{gathered}
\mathbf{a}_{l, m} \approx \frac{4 \pi}{N} !_{i=1}^{N} \mathbf{x}_{i} Y_{l}^{m}\left(\theta_{i}, \phi_{i}\right) \\
\mathbf{x}(\theta, \phi)=!_{l=0}^{H} !_{m=-l}^{l} \mathbf{a}_{l, m} Y_{l}^{m}(\theta, \phi)
\end{gathered}
$$

where $\mathbf{a}_{l, m}=\left[a_{x}, a_{y}, a_{z}\right]^{T}$ is the coefficient vector for level $l$ and order $m$, and $\mathbf{x}_{i}$ $(i=1 \ldots N)$ are the Cartesian coordinates of the object surface. Spherical harmonics are defined as:

$$
Y_{l}^{m}(\theta, \phi)=\sqrt{\frac{2 l+1}{4 \pi} \frac{(l-m) !}{(l+m) !}} P_{l}^{m}(\cos \theta) e^{i m \phi}
$$

where $P_{l}^{m}$ are the associated Legendre polynomials, defined by the differential equation

$$
P_{l}^{m}(x)=\frac{(-1)^{m}}{2^{k} l !}\left(1-x^{2}\right)^{m / 2} \frac{d^{l+m}}{d x^{l+m}}\left(x^{2}-1\right)^{l}
$$

A recursive algorithm for their computation is given in [12]. This yields a parameterization for arbitrary 3D object surfaces of genus zero. The imaginary portion of the series is ignored and only the real portion is used. Note that objects do not have to be star-shaped, as long as $(\theta, \phi)$ is a homologic spherical map. To make the coefficients $\mathbf{a}_{l, m}$ view independent, each ventricle's spherical harmonic coefficients were mapped into a common reference frame by registration using a full-affine transformation.

\subsection{Population Average and BPF Modulation}

The average left lateral ventricle was generated by averaging the coefficients $\mathbf{a}_{l, m}$ of the first 12 harmonics across individuals included in the training set). This average model was then modulated based on the BPF to obtain an approximation of each individual ventricle. The relationship between the BPF and the magnitude of each coefficient of the first 12 harmonics (i.e. $\mathrm{Y}_{1}^{\mathrm{m}}$ with $\mathrm{l}=0, \ldots, 12$, and $\left.\mathrm{m}=-1, \ldots, 0, \ldots, 1\right)$ was tested using linear regression. This process was performed in the $\mathrm{x}, \mathrm{y}$, and $\mathrm{z}$ directions. Coefficients where the $\mathrm{p}$-values for the regression lines were less than or equal to 0.05 were identified as coefficients to modulate and were adjusted based on BPF according to: 


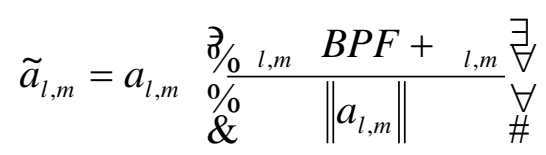

where $a_{l, m}$ is the model parameter of the average ventricle, $\tilde{a}_{l, m}$ is the modulated version approximating the individual shape, and $\beta_{l, m}$ and $\gamma_{l, m}$ are the slope and intercept of the regression line, respectively.

An initial set of customized ventricle approximations was obtained as described above for each of the 31 subjects in the training set. A "leave-one-out" approach was used to ensure that information derived from each test case was not included in the average ventricle. Thus for each case, the customized ventricle was derived from data obtained solely from the other 30 cases considered. New regression lines were determined for each test case and for each spherical harmonic coefficient.

The set of coefficients to include in the approximation model were identified as the coefficients consistently chosen for modulation during the initial "leave-one-out" tests. Thus, the coefficients modulated in at least $90 \%$ of the test cases (i.e. at least 28 of the 31 cases) were determined as being linearly related to BPF and selected as the final set of spherical harmonic coefficients to. This proposed model was also applied to the training set in a "leave-one-out" fashion and further tested on an additional test set of $10 \mathrm{MS}$ patients.

\subsection{Evaluation}

The proposed approximation model was evaluated for each case by comparing both the average ventricle and its individualized modulation to the manual segmentation of the ventricle (gold standard) based on the kappa coefficient, $\kappa$ [13]. The kappa coefficient is a measure of the overlap between two regions and is calculated according to:

$$
\kappa=\frac{2(A \cap B)}{2(A \cap B)+\bar{A} \cap B+A \cap \bar{B}}
$$

where $A$ and $B$ are 3D binary images of the segmented objects, A-bar and B-bar are the inverse images of $\mathrm{A}$ and $\mathrm{B}$, and $\cap$ is the intersection operator (which, in this case, returns the volume of the intersection). Kappa $>=0.7$ indicates excellent agreement. The two kappa coefficients were compared to determine which ventricle (customized or average) represented a closer approximation of the manually segmented ventricle.

\section{Results}

The BPF values measured for the training set and test cases are reported in Table 1 . The BPF values ranged from 0.69 to 0.88 . The mean (s.d.) BPF for the whole group was 0.806 (0.057). The BPF measures obtained for the cases involved in the study was a representative sample of the range of BPF values observed in our laboratory as shown in Figure 1. 
Table 1. Population breakdown

\begin{tabular}{|c|c|c|c|c|}
\hline & Type & $\bar{n}$ & $\mathrm{BPF}$ range & Mean BPF (s.d.) \\
\hline \multirow{4}{*}{ 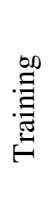 } & Healthy volunteer & 6 & $0.86-0.87$ & $0.87(0.006)$ \\
\hline & Relapsing-remitting MS & 13 & $0.70-0.88$ & $0.82(0.046)$ \\
\hline & Secondary-progressive MS & 9 & $0.69-0.83$ & $0.75(0.043)$ \\
\hline & Primary-progressive MS & 3 & $0.76-0.81$ & $0.78(0.028)$ \\
\hline \multirow{2}{*}{$\begin{array}{l}\overrightarrow{\tilde{\theta}} \\
\stackrel{0}{\Theta}\end{array}$} & Relapsing-remitting MS & 6 & $0.75-0.80$ & $0.77(0.020)$ \\
\hline & Secondary-progressive MS & 4 & $0.70-0.75$ & $0.73(0.023)$ \\
\hline
\end{tabular}
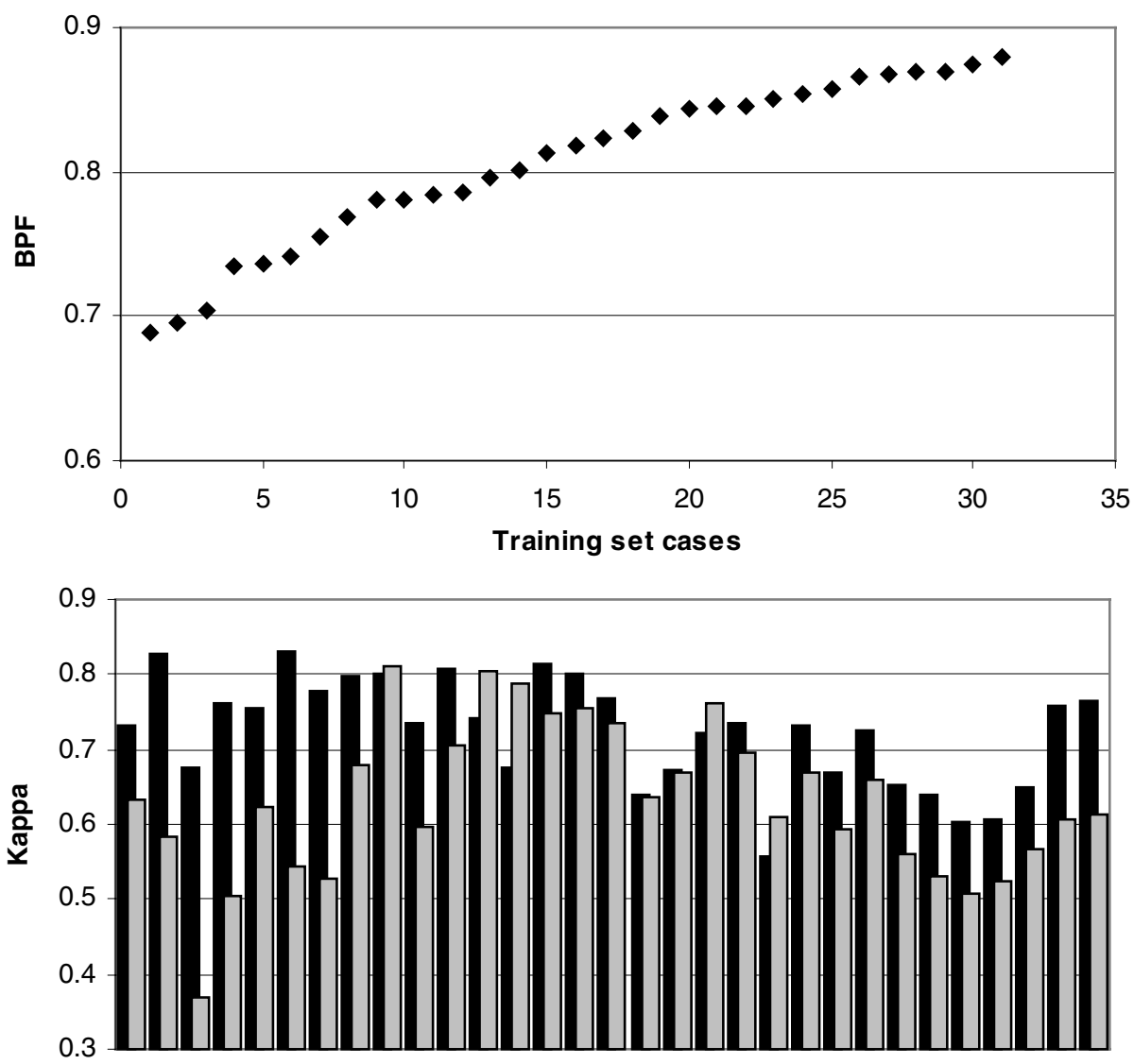

Training set cases

Fig. 1. Top: BPF values of the training set cases. Bottom: Kappa coefficients for the training set population. Kappa coefficient values calculated for each test case by comparing the average ventricle (gray) and customized approximation ventricle (black) to the manually segmented ventricle 
The first $\mathrm{H}=12$ harmonics (eq. 2) were considered for inclusion in the approximation model, which was sufficient to describe the shape of the ventricles. Of the 91 spherical harmonic coefficients tested for modulation in each direction, $34 \%$ (31 of 91 ) were used in the $\mathrm{x}$ direction, $30 \%$ (27 of 91) in the y direction, and $37 \%$ (34 of $91)$ in the $z$ direction.

The "leave-one-out" evaluation of the proposed approximation model on the training set yielded the following results. The mean (s.d.) kappa coefficient for the average ventricles was $0.633(0.103)$ versus 0.721 (0.072) for the customized approximation ventricles, where a kappa coefficient greater than 0.7 indicates excellent agreement. The individual kappa coefficient values for each series are presented in Figure 1. The differences in the kappa coefficients are plotted against BPF in Figure 2. The kappa coefficients calculated from each series were compared using a paired ttest and found to be significantly different $(\mathrm{p}<0.0001)$. Based on the kappa coefficient, the custom approximation represented an improvement over the average ventricle in $81 \%$ of the cases ( 25 of 31 ). Furthermore, $61 \%$ of the custom ventricle (19 of 31 ) had a kappa coefficient larger than 0.7 compared to $26 \%$ of the cases ( 8 of 31 ) for the average ventricle.

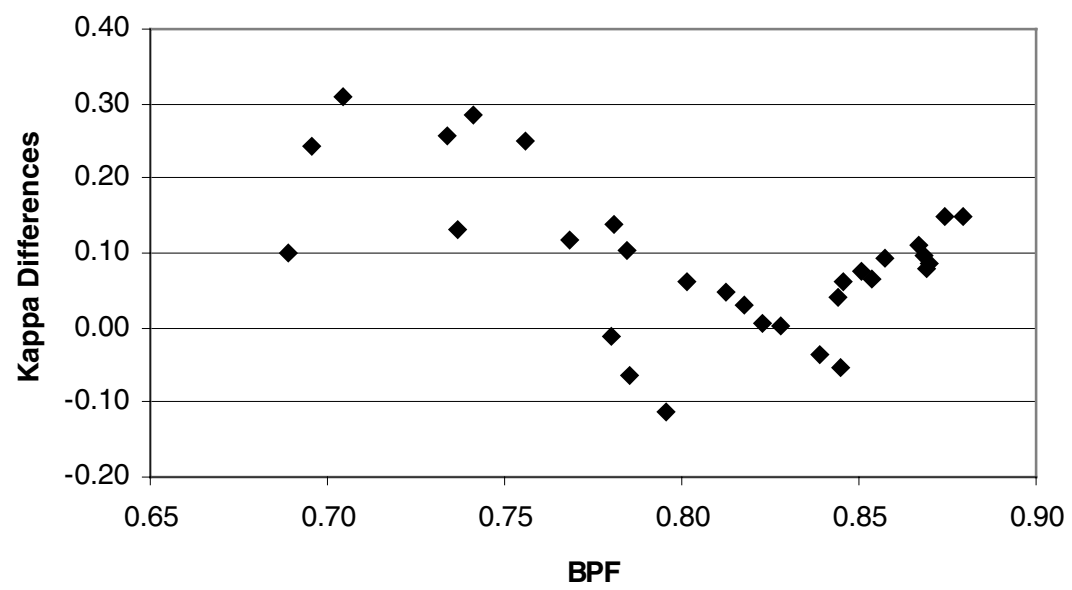

Fig. 2. Differences in Kappa coefficients (customized - average) for the training set cases vs. BPF values

The outcome was similar for the test set with 10 additional MS patients. The mean (s.d.) kappa coefficient for the average ventricles was $0.639(0.098)$ versus 0.796 (0.058) for the customized approximation ventricles, which was also significantly different $(\mathrm{p}<0.001)$. As in the leave-one-out evaluation, the customized ventricle represented a consistent improvement over the average ventricle. The kappa coefficients for the customized approximation were larger than those for the average ventricle in each of the test set cases (Figure 3). In addition, $90 \%$ of the customized ventricles had a kappa coefficient larger than 0.7 , compared to $20 \%$ for the average ventricle.

Figure 4 shows an example axial image with overlaid delineations of the average and customized ventricle boundaries. Figure 5 shows the average and customized ventricle surfaces in 3D for the same patient. 


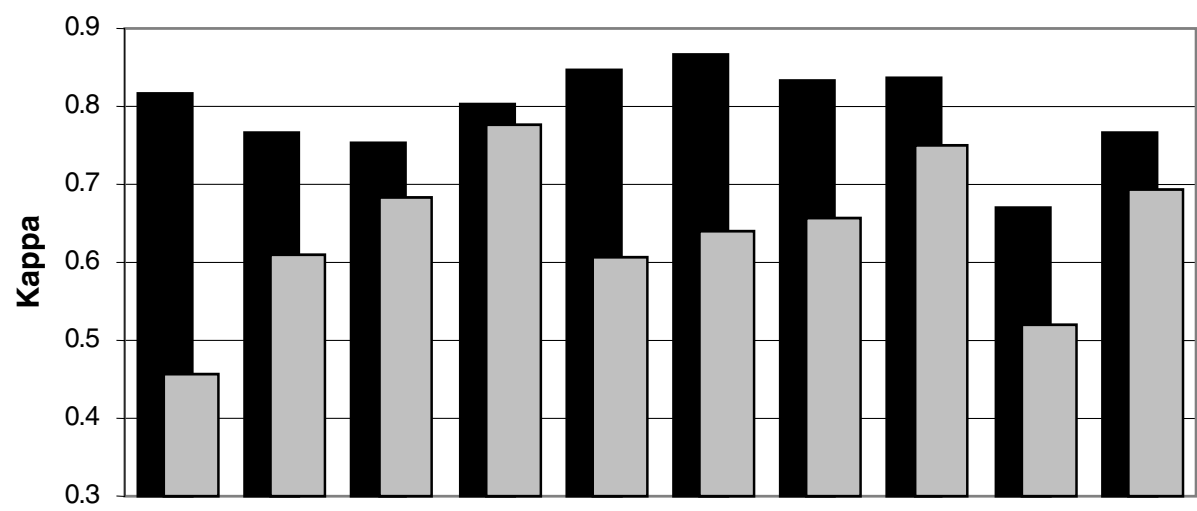

Test set cases

Fig. 3. Kappa coefficients for the test set. Kappa coefficient values calculated for each test case by comparing the average ventricle (gray) and customized approximation ventricle (black) to the manually segmented ventricle

\section{Discussion}

The proposed model reliably and automatically generated customized ventricles based on a population average and BPF. The customized ventricles represented a closer approximation of the target shape than the average ventricle in the majority of the cases ( $81 \%$ and $100 \%$ improvement in the training and test sets, respectively). The improvement was most pronounced for people with BPF significantly different from the mean (see Figure 2), which was expected since the customized ventricles are derived from an average ventricle model. This observation is relevant since a purely statistical model is likely to fail in producing a good instantiation from the same training set for cases with BPF far from the mean. Thus, the proposed approximation model can accommodate any individual case falling within the range of BPFs defined by the training set, regardless of whether similar cases have been observed. Outside of the defined BPF range, the proposed method will rely on extrapolation to approximate expected ventricular shape.

Sensitivity towards the initial condition is an important limitation of deformable models and the main impediment to routine use in clinical morphometry. Statistical priors derived from population averages have been shown to improve optimization efficiency [5]-[7], but an improved approximation of the target shape is likely to further increase both speed and likelihood of convergence to the global minima. The presented model proved especially effective for cases at the tails of the population distribution, where an unmodified statistical prior is likely to fail. Note also that no explicit assumption was made as to the type of deformable model to be used for the segmentation.

The proposed approximation model relies on the assumption of atrophy as a global process and thus relies on a global measure of atrophy and global shape descriptors. Under such conditions, this approach may not perform as well in cases with extreme 
focal atrophy (i.e. very localized shape distortions), unless the same type of focal atrophy is observed in the majority of the training set cases.

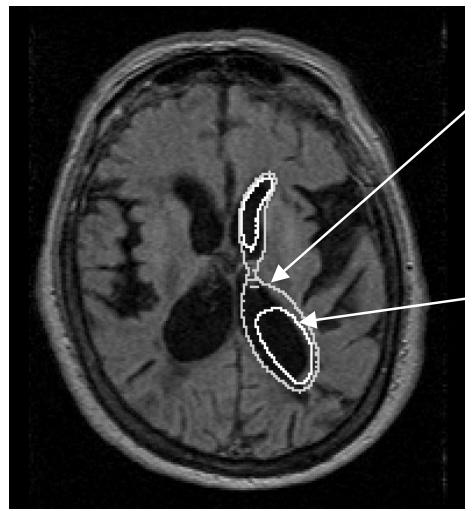

Customized Ventricle

Average ventricle

Fig. 4. Example image with ventricle outlines for the average ventricle and custom approximation of the ventricle

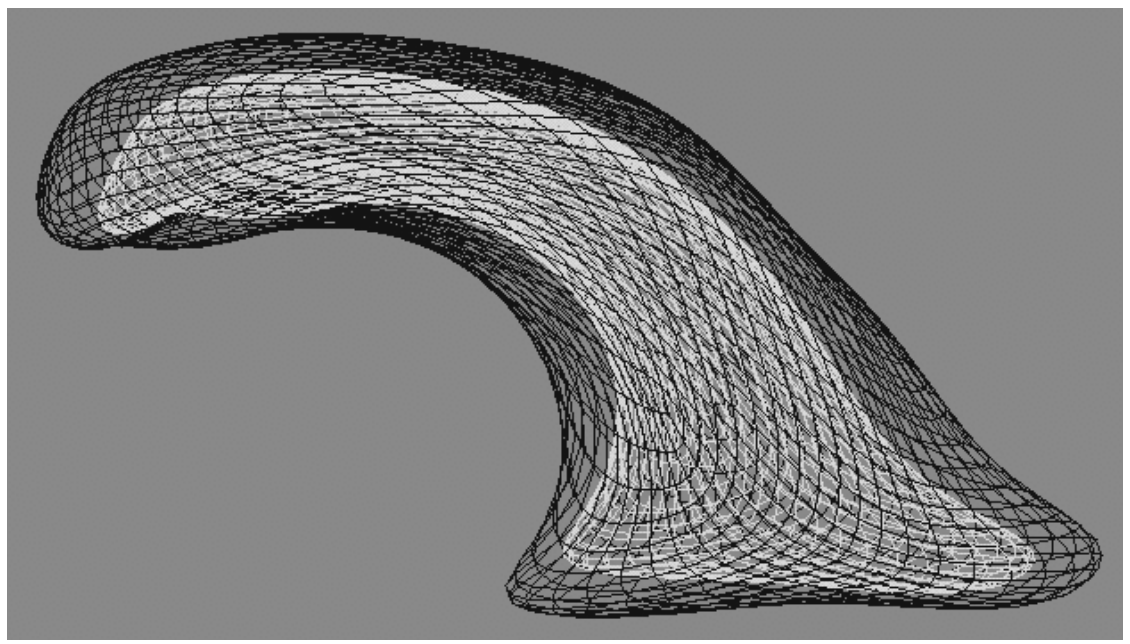

Fig. 5. Approximations of the left ventricular shape. The average ventricular surface (training set) is shown in gray while the customized ventricular surface is shown in black

The new approach was presented on the left ventricle in MS patients. There are, of course, no restrictions as to the right ventricle or other shapes, and further efforts will include additional structures in MS patients and other pathologies. Similar models for Alzheimer's disease and normal aging are of particular interest. Future work will involve applying the custom ventricle as a starting point for automated segmentation of the lateral ventricles in our clinical data. 


\section{Conclusion}

The proposed approximation model was successful in reliably and automatically generating customized ventricles approximating the shape of individual left lateral ventricles. The approximation model relied on a training set consisting of representative MS patients and normal volunteers. The customized approximation of the ventricle was based on the spherical harmonic parameterization of the left ventricle and the associated BPF measures in the training set cases. A set of spherical harmonic coefficients was identified as being linearly related to BPF, and the customized approximation of ventricular shape was generated for a given BPF value by independently modulating the individual coefficients within that set. These customized approximations of the ventricular shape represented a reliably better approximation of the ventricular shape than the average population ventricle (especially for extreme cases), and are, therefore, appropriate for use as a good initial starting point for a variety of segmentation methods.

\section{References}

1. Meier, D.S., Fisher E.: Parameter Space Warping: Shape-Based Correspondence Between Morphologically Different Objects. IEEE Trans. Med. Imag. 21 (2002)

2. Cohen, L.D., Cohen, I.: Finite-Element Methods for Active Contour Models and Balloons for 2D and 3D Images. IEEE Trans Pattern Anal. Mach. Intell. 15 (1993) 1131-1147

3. Klingensmith, J.D., Shekhar, R., Vince, D.G.: Evaluation of three-dimensional segmentation algorithms for the identification of luminal and medial-adventitial borders in intravascular ultrasound images. IEEE Trans. Med. Imag. 19 (2000) 996-1011

4. McInerney, T., Terzopoulos, D.: Deformable Models in Medical Image Analysis: A Survey. Med Imgage Anal. 1 (1996) 91-108

5. Kelemen, A., Szekely, G., Gerig, G.: Elastic Model-Based Segmentation of 3D Neuroradiological Data Sets. IEEE Trans. Med. Imag. 18 (1999) 828-839

6. Staib, L.H., Duncan, J.S.: Model-Based Deformable Surface Finding for Medical Images. IEEE Trans. Med. Imag. 15 (1996) 720-731

7. Cootes, T.F., Taylor, C.J., Cooper, D.H., Graham, J.: Active Shape Models-Their Training and Application. Computer Vision Image Understanding, 61 (1995) 38-59

8. Fisher, E., Cothren, R.M., Tkach, J.A., Masaryck, T.J., Cornhill, J.F.: Knowledge-Based 3D Segmentation of MR Images for Quantitative MS Lesion Tracking. SPIE Med. Imag. 3034 (1997) 599-610

9. Rudick, R., Fisher, E., Lee, J.-C., et al: Use of the Brain Parenchymal Fraction to Measure Whole Brain Atrophy in Relapsing-Remitting MS. Neurology. 53 (1999) 1698-1704

10. Nikou, C. Bueno, G., Heitz, F., Armspach, J.-P.: A Joint Physics-Based Statistical Deformable Model for Multimodality Brain Image Analysis. IEEE Trans. Med. Imag. 20 (2001) 1026-1037

11. Brechbuhler, C., Gerig, G., Kubler, O.: Parametrization of Closed Surfaces dor 3-D Shape Description. Computer Vision Image Understanding, 61 (1995) 154-170

12. Press, W.H., Teutolsky, S.A. Vetterling, W.T., Flannery, B.P.: Numerical Recipes in C the Art of Scientific Computing, $2^{\text {nd }}$ edn. Cambridge University Press, Cambridge (1992)

13. Zijdenbos, A.P., Dawant, B.M., Margolin, R.A., Palmer, A.C.: Morphometric Analysis of White Matter Lesions in MR Images. IEEE Trans. Med. Imag. 13 (1994) 716-724 